repressing our sodium transporters in our kidney, the frequency of salt-sensitive hypertension would have been even higher and hypertension even much more severe.

\section{Acknowledgments}

We thank Jean-Daniel Horisberger, Dmitri Firsov, and Laurent Schild for critically reading the manuscript and Nicole Skarda for secretarial work. This work was supported by a grant from the Swiss National Foundation (no. 31-061966.00 to B.C. Rossier).

1. Guyton, A.C. 1991. Blood pressure control - Special role of the kidneys and body fluid. Science. 252:1813-1816.

2. Lifton, R.P., Gharavi, A.G., and Geller, D.S. 2001. Molecular mechanisms of human hypertension.
Cell. 104:545-556.

3. Achard, J.M., Disse-Nicodeme, S., Fiquet-Kempf B., and Jeunemaitre, X. 2001. Phenotypic and genetic heterogeneity of familial hyperkalaemic hypertension (Gordon syndrome). Clin. Exp. Pharmacol. Physiol. 28:1048-1052.

4. Wilson, F.H., et al. 2001. Human hypertension caused by mutations in WNK kinases. Science. 293:1107-1112

5. Choate, K.A., et al. 2003. WNK1, a kinase mutated in inherited hypertension with hyperkalemia, localizes to diverse Cl-transporting epithelia Proc. Natl. Acad. Sci. U. S. A. 100:663-668.

6. Schambelan, M., Sebastian, A., and Rector, F.C., Jr. 1981. Mineralocorticoid-resistant renal hyperkalemia without salt wasting (Type 2 pseudohypoaldosteronism): role of increased renal chloride reabsorption. Kidney Int. 19:716-727.

7. Yang, C.-L., Angell, J., Mitchell, R., and Ellison, D.H. 2003. WNK kinases regulate thiazidesensitive $\mathrm{Na}-\mathrm{Cl}$ cotransport. J. Clin. Invest. 111:1039-1045. doi:10.1172/JCI200317443.

8. Wilson, F.H., et al. 2003. Molecular pathogenesis of inherited hypertension with hyperkalemia: the $\mathrm{Na}-\mathrm{Cl}$ cotransporter is inhibited by wild-type but not mutant WNK4. Proc. Natl. Acad. Sci. U. S. A 100:680-684.

9. Robert-Nicoud, M., et al. 2001. Transcriptome of a mouse kidney cortical collecting duct cell line: effects of aldosterone and vasopressin. Proc. Natl. Acad. Sci. U. S. A. 98:2712-2716.

10. Nicod, M., et al. 2002. A novel vasopressininduced transcript promotes MAP kinase activation and $\mathrm{ENaC}$ downregulation. EMBO J. 21:5109-5117.

11. Loffing, J., and Kaissling, B. 2003. Sodium and calcium pathways along the mammalian distal nephron: from rabbit to human. Am. J. Physiol. Renal Physiol. In press.

12. Rossier, B.C., Pradervand, S., Schild, L., and Hummler, E. 2002. Epithelial sodium channel and the control of sodium balance: interaction between genetic and environmental factors. Annu. Rev. Physiol. 64:877-897.

13. Kriz, W., and Kaissling, B. 2000. Structural organization of the mammalian kidney. In The kidney: physiology and pathophysiology. 3rd Edition. D.W. Seldin and G. Giebiesch, editors. Lippincot Williams and Wilkins. Philadelphia, Pennsylvania, USA. 587-654.

\title{
Peanut allergy: a growing phenomenon
}

\section{Wesley Burks}

University of Arkansas for Medical Sciences, Arkansas Children's Hospital, Little Rock, Arkansas, USA

J. Clin. Invest. 111:950-952 (2003). doi:10.1172/JCI200318233.

Peanut allergy is one of the most serious of the immediate hypersensitivity reactions to foods in terms of persistence and severity and appears to be a growing problem (1). The prevalence of food hypersensitivity in adults is reportedly less common, but a recent survey in the US found that $1.3 \%$ of adults are allergic to peanuts or tree nuts (2). Recently, in a cohort of American children referred for the evaluation of atopic dermatitis, the prevalence of allergic reactivity to peanuts was nearly twice as high as that in a similar group evaluated a decade earlier (3). In spite of increased recognition and understanding of food allergies, food is the single most common cause

\footnotetext{
Address correspondence to: Wesley Burks, Department of Pediatrics, Division of Allergy and Immunology, Arkansas Children's

Hospital, 1120 Marshall Street, Little Rock, Arkansas 72202, USA.

Phone: (501) 364-1060; Fax: (501) 364-3173;

E-mail: burkswesley@uams.edu.

Conflict of interest: The author owns stock and/or stock options in SEER Inc.
}

of anaphylaxis seen in hospital emergency departments (4), accounting for about one-third of anaphylaxis cases seen. It is estimated that about 30,000 food-induced anaphylactic events are seen in American emergency departments each year, 200 of which are fatal (5). Either peanuts or tree nuts cause more than $80 \%$ of these reactions.

Due to the persistence of the reaction and the lack of effective treatment, peanut-specific immunotherapy is currently being examined as a treatment option. An understanding of the molecular mechanisms is vital to ensure the eventual, effective treatment of peanut-allergic patients.

\section{Th2 polarization of cytokines}

The study by Turcanu et al. (6) in this issue of the JCI uses a novel approach, that of CFSE staining to separate peanut-specific lymphocytes by flow cytometry and subsequent cloning. Due to technical difficulties arising from the low frequency of allergenspecific cells in the blood, previous studies have been complicated and not easily interpreted. Human T cells, when repeatedly stimulated in vitro, will often develop a Th2 phenotype regardless of their origin $(7,8)$.

In this study, the authors compare peanut-allergic individuals, individuals who have outgrown peanut allergy, and those who had always tolerated peanuts (6). Peripheral blood lymphocytes in the peanut-allergic individuals demonstrated a Th2 polarization of cytokine production by peanut-specific cells with low levels of IFN- $\gamma$ and TNF- $\alpha$ and high levels of IL-4, IL-5, and IL-13. In the individuals who had outgrown their peanut allergy and the nonallergic controls, the cytokines showed a Th1 bias with high levels of IFN- $\gamma$ and TNF- $\alpha$ and low levels of IL-4, IL-5, and IL-13. An interesting part of the study investigated individuals who were allergic to eggs, using similar laboratory and cloning techniques. As in the peanutspecific cells, the egg-allergic individuals' peripheral blood lymphocytes had a Th2 skewing of their ovalbumin-specific T cells (Figure 1).

This evidence that peanut antigens do not in themselves induce a Th2 cytokine response is not surprising, since all food allergy patients share similar clinical symptoms in the skin, gastrointestinal tract, and respiratory tract. The results of the present study (6) will help in the evaluation of future immunomodulatory treatments for food allergy and in studies 


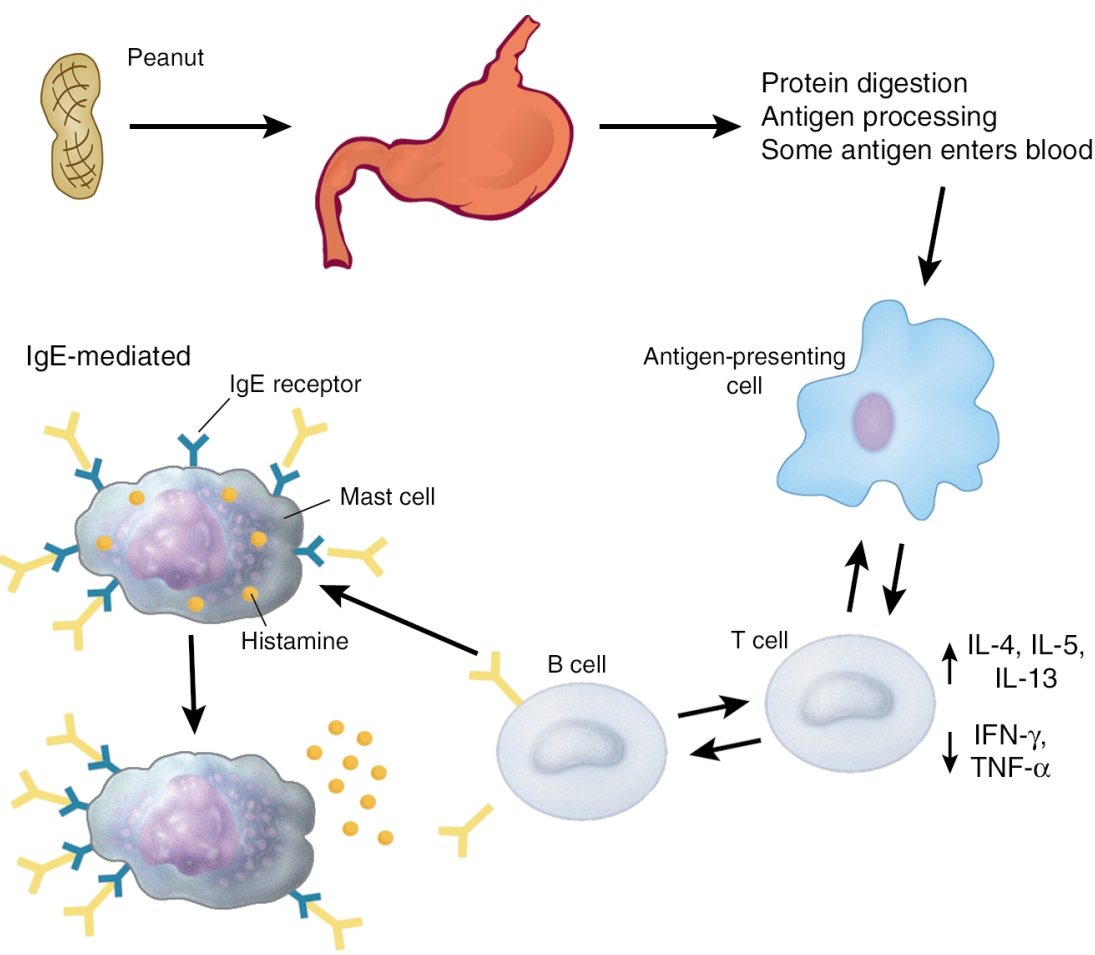

Figure 1

Foods enter the gastrointestinal tract and undergo protein digestion and then antigen $(\mathrm{Ag})$ processing. Some Ag enters the blood stream to be distributed at distal sites throughout the body (particularly the skin and respiratory mucosa along with sites in the gastrointestinal mucosa). As the antigen-presenting cell presents $\mathrm{Ag}$ to the $\mathrm{T}$ cell, specific cytokines are produced. In the peanutallergic individual, the T cells will secrete increased amounts of IL-4, IL-5, and IL-13, among other mediators, and reduced amounts of IFN- $\gamma$ and TNF- $\alpha$ when compared to that in an individual who is not allergic to peanuts. The $T$ cell in turn regulates eventual peanut-specific IgE production by $B$ cells. Peanut-specific IgE is attached to mast cells in the gastrointestinal tract, skin, and respiratory tract mucosa. Subsequently, on ingestion of peanuts, in the peanut-allergic individual, the protein is digested and the Ag binds peanut-specific IgE on the mast cell, causing activation of the mast cell with mediator release at the mucosal site. Clinical symptoms ensue.

that examine the natural history of food allergic reactions.

\section{Peanut-specific IgE}

The sequence of events necessary to produce allergen-specific IgE suggests several potential avenues for immunomodulatory intervention. These approaches can be placed into two general categories: (a) strategies designed to inhibit $\operatorname{IgE}$ or the interaction of mediators important to IgE production, and (b) strategies designed to alter $T$ cell activation. The second category includes induction of $\mathrm{T}$ cell anergy by use of allergen-specific peptides ( $T$ cell epitopes), and modulation of Th cell development by means that favor a Th1 cytokine response and/or by prevention of $\mathrm{T}$ cell proliferation.

Allergen immunotherapy is an effective therapeutic modality to prevent further anaphylactic episodes in mechanism of the food hypersensitivity response that can be studied as patients with food allergy are placed on food-specific immunotherapy.

One of the more exciting recent developments in the treatment of food allergy is novel immunotherapeutic strategies designed to alter the immune system's response to food allergens. These strategies are now being examined in animal models as potential treatment modalities (11). They include cytokine-modulated immunotherapy, immunostimulatory sequence-modulated immunotherapy, plasmid DNA immunotherapy, allergen-peptide immunotherapy, and "engineered" (mutated) allergen protein immunotherapy. All of these methods strive to elicit a Th1-type response or tolerance from the immune system in response to a specific food allergen.

\section{Natural history of peanut allergy}

It will be even be more interesting to elucidate why only a few food allergens induce the type of Th2 response early in life that is demonstrated in this study (6), and why certain people outgrow their food allergy. In this study, individuals allergic to peanuts and individuals allergic to eggs had a similar Th2-skewed cytokine response. However, the natural history of the clinical symptoms of peanut allergy is quite different from that of the symptoms of egg allergy. Peanut allergy is infrequently outgrown (12), while egg allergy is typically outgrown in the first 4-5 years of life.

The novel approach used by these authors to identify antigen-specific $\mathrm{T}$ cells will likely have important implications for future studies of allergic disease, as well as autoimmune disorders. allergens, it is now being studied as a possible option for patients with lifelong food allergies (10). The goal of peanut-specific immunotherapy would not be to allow patients to eat peanuts at will, but rather to give them some protection in case of accidental ingestion. Given the partial rate of response and high rate of adverse reactions to traditional food immunotherapy in previous studies, and the frequent occurrence of accidental peanut ingestion, alternative forms of immunotherapy are necessary for this potentially fatal allergy. The findings by Turcanu et al. (6) provide additional laboratory data about the possible
1. Sampson, H.A. 2002. Clinical practice. Peanut allergy. N. Engl. J. Med. 346:1294-1299.

2. Sicherer, S.H., Munoz-Furlong, A., Burks, A.W. and Sampson, H.A. 1999. Prevalence of peanut and tree nut allergy in the US determined by a random digit dial telephone survey. J. Allergy Clin. Immunol. 103:559-562.

3. Sampson, H.A. 1996. Managing peanut allergy. BMJ. 312:1050-1051.

4. Yocum, M.W., et al. 1999. Epidemiology of anaphylaxis in Olmsted County: a populationbased study. J. Allergy Clin. Immunol. 104:452-456.

5. Bock, S.A., Munoz-Furlong, A., and Sampson, H.A. 2001. Fatalities due to anaphylactic reactions to foods. J. Allergy Clin. Immunol. 107:191-193.

6. Turcanu, V., Maleki, S.J., and Lack, G. 2003. Characterization of lymphocyte responses to peanuts in normal children, peanut-allergic chil- 
dren, and allergic children who acquired tolerance to peanuts. J. Clin. Invest. 111:1065-1072. doi:10.1172/JCI200316142.

7. Cohen, S.B., Webb, L.M., and Feldmann, M 1996. The method of deriving human T-cell clones alters the proportion of IL-10-producing cells. Immunology. 87:343-347.

8. Demeure, C.E., et al. 1995. Human naive CD4 T cells produce interleukin-4 at priming and acquire a Th2 phenotype upon repetitive stimulations in neutral conditions. Eur. J. Immunol. 25:2722-2725.

9. Valentine, M.D., et al. 1990. The value of immunotherapy with venom in children with allergy to insect stings. N. Engl. J. Med. 323:1601-1603

10. Oppenheimer, J.J., Nelson, H.S., Bock, S.A., Christensen, F., and Leung, D.Y. 1992. Treat- ment of peanut allergy with rush immunotherapy. J. Allergy Clin. Immunol. 90:256-262.

11. Burks, A.W., Bannon, G.A., and Lehrer, S.B. 2001. Classic specific immunotherapy and new perspectives in specific immunotherapy for food allergy. Allergy. 67:121-124.

12. Skolnick, H.S., et al. 2001. The natural history of peanut allergy. J. Allergy Clin. Immunol. 107:367-374.

\title{
Heparan sulfate: Antithrombotic or not?
}

\author{
Jeffrey I. Weitz \\ Henderson Research Centre, Hamilton, Ontario, Canada \\ J. Clin. Invest. 111:952-954 (2003) doi:10.1172/JCI200318234.
}

Tissue factor exposed at sites of vascular injury initiates coagulation resulting in thrombin generation. In addition to converting fibrinogen to fibrin, thrombin amplifies its own generation by activating platelets and by activating factors $\mathrm{V}$ and VIII, key cofactors in coagulation. Consequently, tight regulation of thrombin activity is essential to prevent excessive thrombosis (1).

\section{Regulation of thrombin}

Two naturally occurring anticoagulant pathways serve to regulate thrombin; the protein $\mathrm{C}$ pathway and antithrombin. The protein $C$ pathway is initiated when thrombin binds to thrombomodulin, a thrombin receptor expressed by endothelial cells (2). Once bound to thrombomodulin, thrombin undergoes a conformational change at its active site that converts it from a procoagulant enzyme into a potent activator of protein C. Activated protein $\mathrm{C}$, in concert with its cofactor protein $S$, serves as an anticoagulant by degrading and inactivating activated factors V and VIII (2).

Several lines of evidence highlight the physiological importance of the

\footnotetext{
Address correspondence to: Jeffrey I. Weitz, Henderson Research Centre, 711 Concession Street, Hamilton, Ontario L8V 1C3, Canada. Phone: (905) 574-8550; Fax: (905) 575-2646; E-mail: jweitz@thrombosis.hhscr.org. Conflict of interest: The author has declared that no conflict of interest exists.

Nonstandard abbreviations used: serine proteinase inhibitor (serpin); 3-O-sulfotransferase-1 (3-OST-1).
}

protein $\mathrm{C}$ pathway. Mice totally deficient in thrombomodulin die in utero (3), whereas ablation of endothelial thrombomodulin causes early onset thrombosis (4). Likewise, patients with protein $\mathrm{C}$ or protein $\mathrm{S}$ deficiency are prone to thrombosis, as are those with the factor $V$ Leiden mutation, a point mutation that renders activated factor $\mathrm{V}$ Leiden relatively resistant to inactivation by activated protein $C(5)$.

Antithrombin also is critical for the regulation of coagulation. A member of the serine proteinase inhibitor (serpin) superfamily, antithrombin inhibits thrombin and other clotting enzymes in a slow, progressive fashion. The importance of antithrombin is highlighted by the fact that patients with heterozygous antithrombin deficiency have a thrombotic tendency $(5,6)$. Complete deficiency of antithrombin is likely to be incompatible with life, a concept supported by the observation that knocking out the antithrombin gene in mice results in intrauterine death from massive thrombosis (7).

\section{Heparan sulfate}

The activity of antithrombin is enhanced by heparin. Antithrombin possesses a heparin binding site that interacts with a unique pentasaccharide sequence found on one-third of the chains of unfractionated heparin. Key to high-affinity binding of antithrombin to this pentasaccharide sequence is 3-O-sulfated glucosamine, the middle saccharide unit of the pentasaccharide (8). Pentasaccharide binding to antithrombin induces conformational changes in the reactive center loop of the serpin that accelerate the rate at which antithrombin inhibits its target proteases by two to three orders of magnitude.

Although heparin is found in granules of human mast cells, it is not the physiological counterpart of medicinal heparin. Recent studies indicate that mast cell heparin regulates the types and amounts of positively-charged proteases stored in mast cell granules. Thus, mast cells deficient in heparinsynthesizing enzyme have altered morphology (9) and their granules contain reduced amounts of tryptase, chymase, and carboxypeptidase A (10). Not only are protease levels reduced, but their activity may also be limited because heparin enhances the activity of some mast cell proteases (11).

Current thinking is that the physiological counterpart of medicinal heparin is heparan sulfate, a glycosaminoglycan found on the surface of most eukaryotic cells and in the extracellular matrix. Anchored to the cell surface by its proteoglycan core, the functional units of heparan sulfate are found on its branching glycosaminoglycan side-chains (Figure 1). Cultured endothelial cells synthesize heparan sulfate and $1 \%$ to $10 \%$ of the molecules have anticoagulant activity because they contain the 3-O-sulfated glucosamine residue that is the hallmark of the antithrombin-binding pentasaccharide (11).

Antithrombin binds to cultured endothelial cells and binding is reduced when the cells are pretreated with heparinase (12), suggesting that this interaction is mediated by heparan sulfate. When radiolabeled antithrombin is used, over $90 \%$ of the antithrombin that binds to cultured endothelial cells or to the surface of perfused aortic segments can be localized to the subendothelial matrix (13). Exposure of cultured endothelial cells to IL-1 or tumor necrosis factor reduces heparan sulfate 